\title{
Localization of cortactin is associated with colorectal cancer development
}

\author{
HIROSHI HIRAKAWA ${ }^{1}$, KENICHIRO SHIBATA ${ }^{1,2}$ and TOSHIYUKI NAKAYAMA ${ }^{1}$
}

\author{
${ }^{1}$ Department of Tumor and Diagnostic Pathology, Atomic Bomb Disease Institute, Nagasaki University Graduate School of \\ Biomedical Sciences, 1-12-4 Sakamoto, Nagasaki 852-8523; ${ }^{2}$ Division of Surgical Oncology, Department of Translational \\ Medical Sciences, Nagasaki University Graduate School of Biomedical Sciences, 1-7-1 Sakamoto, Nagasaki 852-8501, Japan
}

Received August 21, 2009; Accepted September 22, 2009

DOI: 10.3892/ijo_00000444

\begin{abstract}
Cortactin is a ubiquitously expressed actin filament (F-actin)-binding protein that stabilizes F-actin networks and promotes actin polymerization by activating the actinrelated protein 2/3 (Arp2/3) complex. Overexpression of cortactin in cancer cells stimulate cell migration, invasion, and experimental metastasis; however, the underlying mechanism in cortactin involvement in tumor progression is not fully understood. Recently, a direct interaction between zonula occludens-1 (ZO-1) and cortactin in epithelial cells was reported. The present study aimed to further clarify the significance of the interaction between cortactin and $\mathrm{ZO}-1$ in cancer progression. Cortactin expression and localization in colorectal human cancer tissues were evaluated by immunohistochemistry and immunofluorescence. Co-immunoprecipitation and immunofluorescence analysis revealed cortactin and ZO-1 interaction and localization in cancer cells. In our study, the localization of cortactin is a crucial marker for lymph node metastasis in colorectal cancer. We show how the localization of cortactin effects cancer development. A molecular interaction between cortactin and ZO-1 in migrating or polarized cancer cells was revealed. This is the first report to show the interaction of cortactin and ZO-1 in colorectal cancer progression. We conclude that localization of cortactin in cancer cells and interaction between ZO-1 and cortactin are crucial for cancer progression.
\end{abstract}

\section{Introduction}

Cortactin is an actin filament (F-actin)-binding protein that localize at the cortical regions of cells and is a prominent substrate for Src-family protein tyrosine kinases in response

Correspondence to: Dr Hiroshi Hirakawa, Department of Tumor and Diagnostic Pathology, Atomic Bomb Disease Institute, Nagasaki University Graduate School of Biomedical Sciences, 1-12-4 Sakamoto, Nagasaki 852-8523, Japan

E-mail: h-hira@nagasaki-u.ac.jp

Key words: cortactin, ZO-1, immunohistochemistry, cancer development, colon to multiple extracellular stimuli $(1,2)$. A human homolog was identified as a protein product of a putative oncogene, EMS1, and has been implicated in both cell adhesion and cell signaling (3). Cortactin is encoded by the CTTN gene and maps to chromosome 11q13 (4). The N-terminal half of the protein includes the N-terminal acidic region (NTA), the binding site for actin-related protein 3 (Arp3) (a component of the Arp2/3 complex) followed by 6.5 tandem repeats of 37 amino acids responsible for $\mathrm{F}$-actin binding (5). The $\mathrm{C}$-terminal half is composed of an $\alpha$-helix domain of unknown function, followed by a proline-serine-threonine-rich region (PST) and Src homo$\operatorname{logy} 3$ (SH3) domain (6). Gene amplification of CTTN and overexpression of cortactin have been reported in various cancers including colorectal cancer (7-11).

Cortactin binds to and potentiates the activity of Arp2/3 (12-14), which drives the remodeling of the actin cytoskeleton (15). Remodeling of the actin cytoskeleton is essential for cell migration, cell adhesion, and cell polarization as well as for tumor cell invasion and metastasis. Indeed, cells overexpressing cortactin show enhanced cell migration and invasion $(16,17)$ and increased metastatic potential in mice $(18)$.

Zonula occludens-1 (ZO-1) is an F-actin-binding protein, containing three amino-terminal PSD95/dlg/ZO-1 (PDZ) domains, followed by an SH3 domain, a guanylate kinase (GK) domain, and a proline-rich region in this order forming the N-terminus $(19,20)$. In polarized epithelial cells, ZO-1 exclusively localizes to a tight junction (TJ) and directly binds to claudin at the first PDZ domain (21), occludin at the GK domain (22), and F-actin at the C-terminal half and connects claudin to the actin cytoskeleton $(22,23)$.

The cortactin SH3 domains have been shown to bind to the proline-rich region of ZO-1 (24). However, very little is known about the function of $\mathrm{ZO}-1 /$ cortactin complex. In the present study, we show an association between cortactin localization in human colorectal cancer cells and lymph node metastasis. We also show interaction between $\mathrm{ZO}-1$ and cortactin at TJ. The association of ZO-1 and cortactin may directly play important roles in the formation and/or regulation of cell-cell adhesion and tumor progression.

\section{Materials and methods}

Antibodies. Mouse monoclonal anti-ZO-1 (1:100; Lifespan Biosciences, Seattle, WA, USA), anti-E-cadherin (1:250; 
Dako, Glostrup, Denmark) and anti- $\beta$-catenin $(1: 250 ; \mathrm{BD}$ Bioscience, San Jose, CA, USA) antibodies were used for the immunodetection of proteins. Rabbit polyclonal anti-cortactin (1:100; Genway Biotech, San Diego, CA, USA), anti-ß-catenin (1:250; Thermo Scientific, Waltham, MA, USA) and antiZO-1 (1:250; LifeSpan Biosciences) antibodies were used.

Cell culture. LS123 (human colon cancer) cells, kindly provided by Dr T. Nakayama (Atomic Bomb Disease Institute, Nagasaki, Japan), were grown in minimal essential medium (MEM, Gibco Invitrogen, Carlsbad, CA, USA) supplemented with penicillin/streptomycin plus $10 \%$ fetal calf serum (FCS) at $37^{\circ} \mathrm{C}$ in $5 \% \mathrm{CO}_{2}$. Cells of $30 \%$ confluence and $100 \%$ confluence in a $10-\mathrm{cm}$ plastic dish were used for immunoprecipitation study. Cell with $70 \%$ confluency were used for immunocytochemical analysis.

Immunohistochemistry. We randomly chose samples from surgical specimens that were subjected to pathological diagnoses in our institution from 2005 to 2008. Forty colorectal cancer cases were studied. In previous reports, cortactin expression was related to depth of invasion (10). To reveal the association of cortactin expression with cancer cell metastasis, we randomly selected $\mathrm{T} 2$ stage according to the TNM classification system. All investigations were approved by our institutional review board, and informed consent was obtained from all participants prior to the study. We prepared 4- $\mu$ m-thick sections from formalin-fixed, paraffin-embedded tissue blocks and subjected them to immunohistochemical analyses with an avidin-biotin complex detection system (Vectastain; Vector Laboratories, Burlingame, CA, USA). To facilitate antigen retrieval, the sections were incubated in Tris-ethylenediaminetetraacetic acid (EDTA) buffer ( $\mathrm{pH} 9.0$ ) at $121^{\circ} \mathrm{C}$ for $15 \mathrm{~min}$. Images were captured with a Nikon Digital Sight Fi1 camera (Nikon, Tokyo, Japan). A diaminobenzidine (DAB) kit (Histofine; Nichirei, Tokyo, Japan) was used for the detection of immunoreactions. The control sections were incubated with isotype-matched immunoglobulin (Ig) G.

Immunocytochemistry. Cells were fixed with 4\% paraformaldehyde, permeabilized with $0.25 \%$ Triton X-100, and processed for immunofluorescence analysis as described below.

Immunofluorescence analysis. After incubation with primary antibodies, the sections were further incubated with secondary antibodies (Alexa 488 conjugated affinipure goat anti-mouse IgG and Alexa 555 conjugated affinipure goat anti-rabbit IgG) obtained from Molecular Probes (Eugene, OR, USA). Slides were then mounted with Vectashield mounting medium containing 4,6-diamidino-2-phenylindole (DAPI) nuclear counterstain (Vector Laboratories). The fluorescence images were examined using an MD2500 microscope (Leica Microsystems, Tokyo, Japan) with x40 and x100 objective lens. The pictures were captured with a Leica DC500 camera (Leica Microsystems).

Immunoprecipitation analysis. Cells were incubated in ice-cold lysis buffer consisting $50 \mathrm{mM}$ Tris- $\mathrm{Cl}$ (pH 7.6), $150 \mathrm{mM} \mathrm{NaCl}$,
$0.1 \%$ Nonidet P-40, and 1 tablet of Complete protease inhibitor cocktail (Roche Diagnostics, Indianapolis, IN) and incubated on ice for $30 \mathrm{~min}$. Lysates were cleared by centrifugation at $15,000 \mathrm{rpm}$ for $20 \mathrm{~min}$. The supernatant was precleared by incubating with Protein A/G Plus Agarose beads (Thermo Scientific) for $1 \mathrm{~h}$ at $4^{\circ} \mathrm{C}$. Four hundred microliters $(200 \mu \mathrm{g}$ of total protein) of precleared extracts were incubated with 2-5 $\mu 1$ of monoclonal anti-ZO-1, anti- $\beta$-catenin or rabbit polyclonal anti-cortactin antibodies at $4^{\circ} \mathrm{C}$ for $1 \mathrm{~h}$. Immune complexes were recovered by adding $40 \mathrm{ml}$ of Protein $\mathrm{A} / \mathrm{G}$ Plus Agarose beads, following by incubation at $4^{\circ} \mathrm{C}$ for $1 \mathrm{~h}$. The beads were washed three times with PBS, boiled in SDS sample buffer, and processed for immunoblotting as described below.

Gel electrophoresis and immunoblotting. The immunoprecipitates were separated by sodium dodecyl sulfate-polyacrylamide gel electrophoresis (SDS-PAGE) and the proteins were then transferred to Immobilon-P membranes (Millipore, Billerica, MA, USA). The membranes were blocked with $5 \%$ non-fat dry milk in phosphate-buffered saline (PBS) containing $0.05 \%$ Tween-20 and then incubated with rabbit polyclonal anti-ZO-1 antibody (1:100), anti-ß-catenin (1/500) or anti-cortactin antibody (1:100). Reactivity was detected by the using horseradish peroxidase-conjugated secondary antibodies and chemiluminescence (ECL, Amersham Pharmaceuticals, Amersham, UK).

Evaluation and statistical analysis. Cortactin staining was considered to be 'overexpression' if $>50 \%$ of cancer cells showed immunoreactivity in the cytoplasm diffusely or at the apical region, and to be 'periphery' if $>20 \%$ of cancer cells at the invasive site showed immunoreactivity at the peripheral (membranous) region by immunohistochemistry. The correlations between the staining pattern and positivity of lymph node metastasis were calculated by using the $\chi^{2}$ test at a $5 \%$ significance level.

\section{Results}

Localization of cortactin in normal colon epithelium and colorectal cancer. Immunoreactivity of cortactin was found at the apical border of normal colon epithelium where it colocalized with ZO-1. In colorectal cancer, the localization of cortactin was often separated from ZO-1. In cancer cells, immunoreactivity of cortactin was not only found at the apical region but also often observed at the cell periphery (Fig. 1).

Localization of ZO-1 and E-cadherin. Apical localization of ZO-1 (Fig. 2A) and basolateral localization of E-cadherin (Fig. 2C) was observed in the normal epithelium. Cells of some components of poorly differentiated adenocarcinoma occasionally showed some polarization. In those cells, apically ZO-1 (Fig. 2B) and basolaterally E-cadherin (Fig. 2D) were found. In depolarized cells in poorly differentiated adenocarcinoma, spot-like cytoplasmic localization of Ecadherin was found in disseminated cancer cells (Fig. 2D). Spot-like and/or membranous localization of ZO-1 in poorly differentiated adenocarcinoma was detected in disseminating cells (Fig. 2B) (25). We could not reveal a clear relationship 

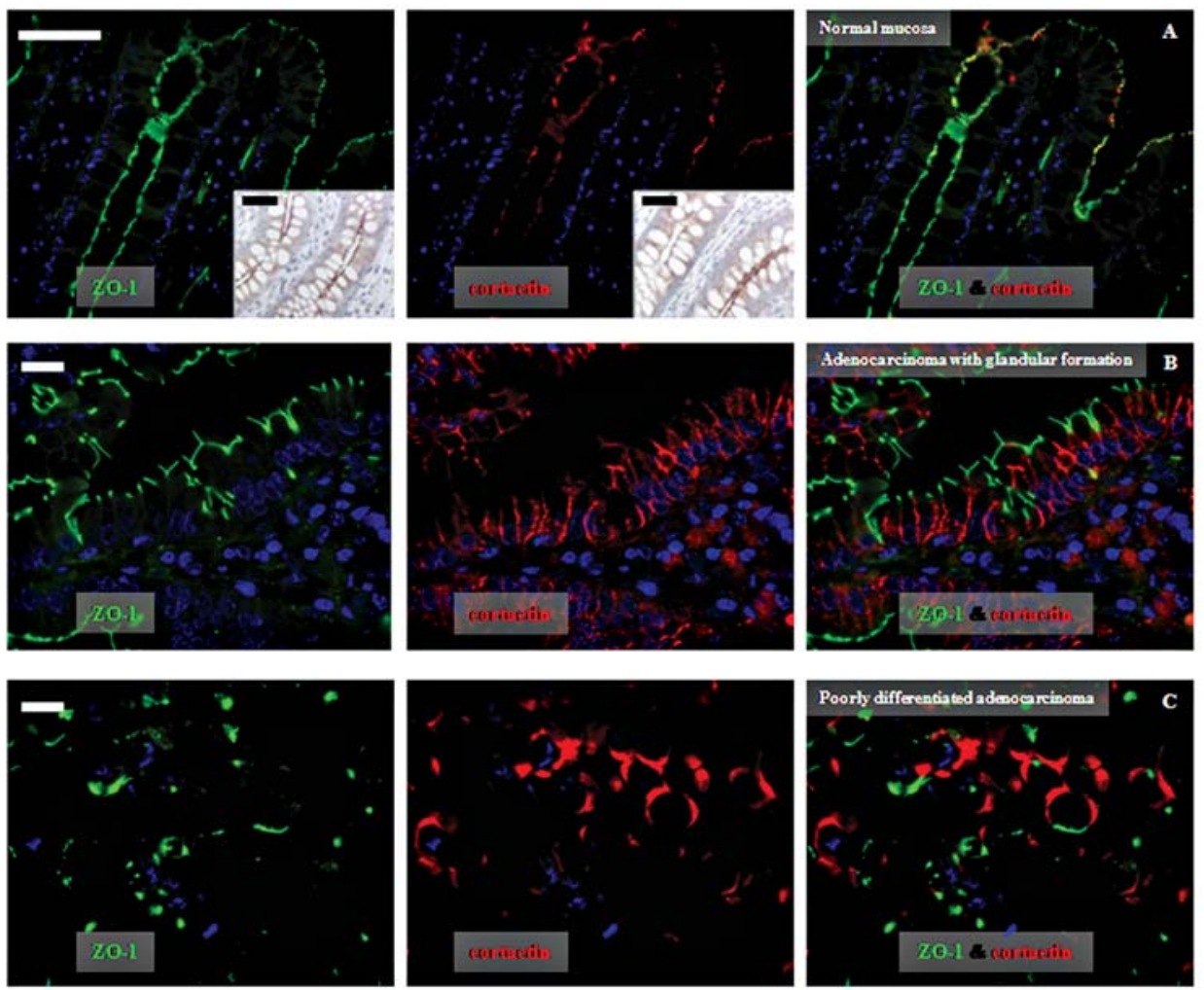

Figure 1. Localization of cortactin in normal colon epithelium (A) and colorectal cancer (B and C). (A) Localization of ZO-1 (green, left panel) and cortatin (red, middle panel). Colocalization of ZO-1 and cortactin was found in normal colon epithelium. Bar, $50 \mu \mathrm{m}$. (B and C) Localization of ZO-1 (green) and cortactin (red) in adenocarcinoma with glandular formation. (B) Bar, $20 \mu \mathrm{m}$ and poorly differentiated adenocarcinoma. (C) Bar, $10 \mu \mathrm{m}$. ZO-1 and cortactin was separately distributed in cancer cells.
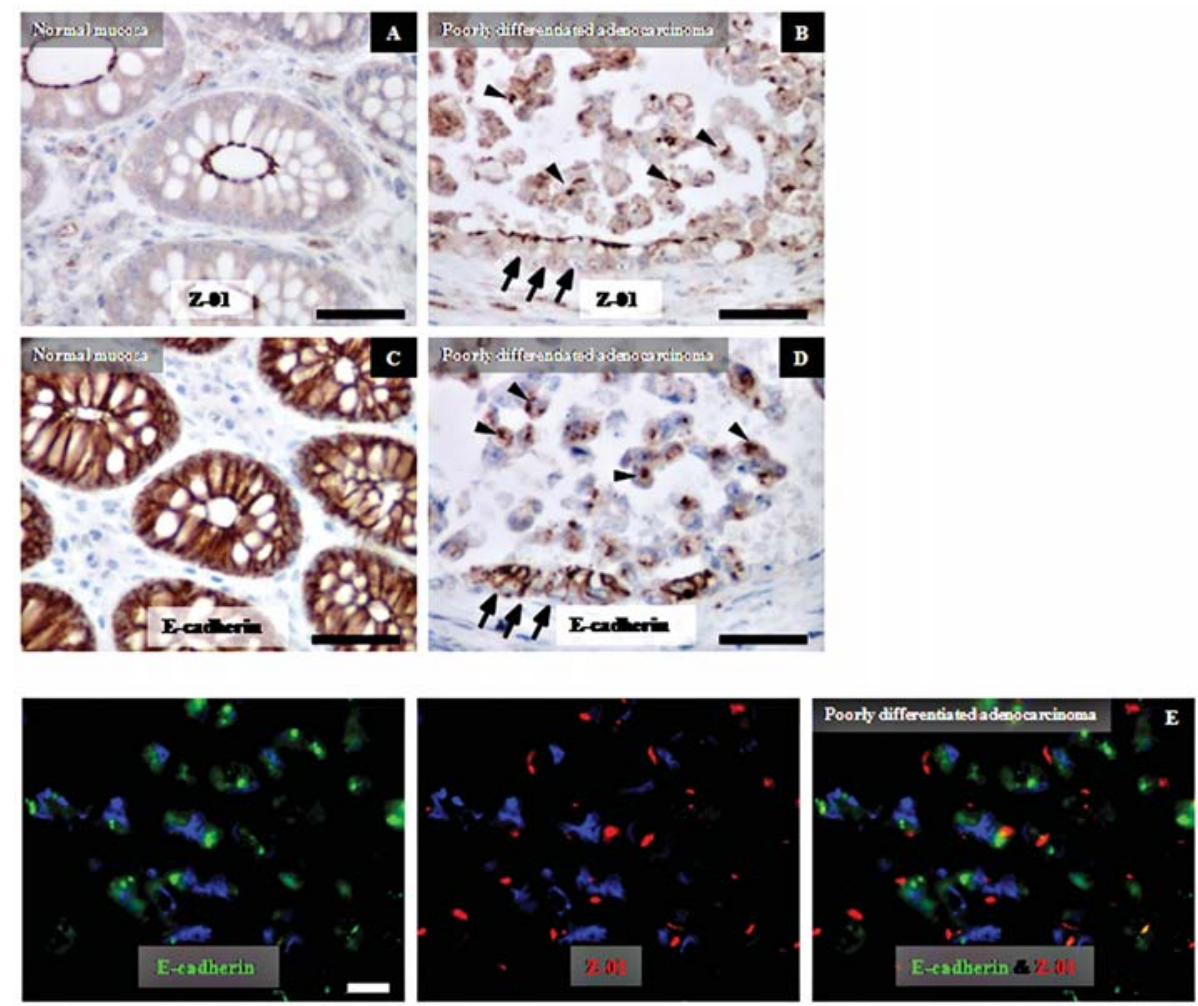

Figure 2. Localization of ZO-1 and E-cadherin in normal colon epithelium (A and C) and colorectal cancer (B, D and E). (B and D) Serial sections. (A) Apical localization of ZO-1 was found in normal epithelium. (C) Basolateral localization of E-cadherin was found in normal epithelium. (B) Apically ZO-1 was detected in the cancer cells, which were somewhat polarized (arrows). Spot-like and/or membranous localization of ZO-1 in poorly differentiated adenocarcinoma was detected in disseminating cells (arrowheads). (D) Basolaterally E-cadherin was detected in polarized cells (arrows). In depolarized cells in poorly differentiated adenocarcinoma, spot-like localization of E-cadherin in the cytoplasm was found (arrowheads). Bar, $50 \mu \mathrm{m}$. (E) ZO-1 (green, left panel) and E-cadherin (red, middle panel) in cancer cells with immunofluorescence. No clear relationship of localization of ZO-1 and E-cadherin was found. Bar, $10 \mu \mathrm{m}$. 

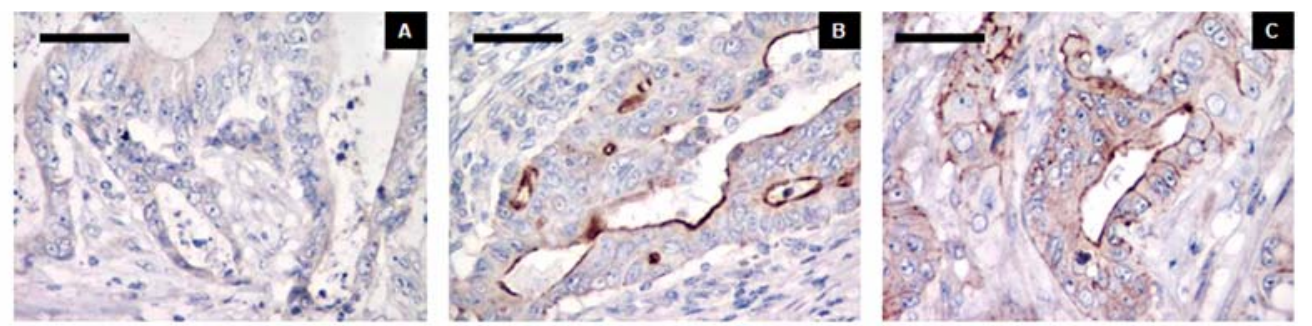

Figure 3. Representative staining pattern of cortactin. (A) Immunoreactivity of cortactin was not detectable. (B) Immunoreactivity of cortactin was detectable only at the apical border. (C) Cortactin was localized at peripheral (membranous) region of cancer cells. Bar, $50 \mu \mathrm{m}$.

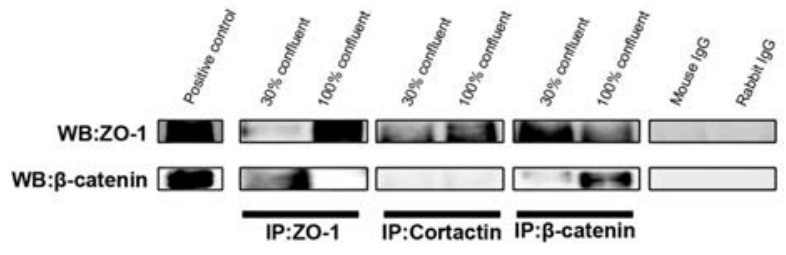

Figure 4. Co-immunoprecipitation of ZO-1/cortactin and ZO-1/ß-catenin in $30 \%$ confluent and $100 \%$ confluent LS123 cells. Higher interaction of ZO-1 and cortactin was found in $100 \%$ confluent cells and lower interaction of these molecules was observed in $30 \%$ confluent cells. Lower interaction of ZO-1 and B-catenin was found in $100 \%$ confluent cells and lower interaction of these molecules was obtained in $30 \%$ confluent cells. In this study, immunoprecipitant of cortactin was not found immunoprecipitated with $\beta$-catenin. Isotope matched mouse and rabbit IgGs were used as negative controls. of localization of ZO-1 and E-cadherin in cancer cells with immunofluorescence studies (Fig. 2E).

Association of cortacin immunostaining pattern and lymph node metastasis. Immunoreactivity for cortacin was observed in the cytoplasm and/or the apical side of glandular formations and/or periphery of cancer cells. Representative staining pattern of cortactin is shown in Fig. 3. Cortactin was detected by immunohistochemistry in 25 of the 40 patients $(62.5 \%)$ examined. A relationship between cortactin overexpression and lymph node metastasis was not observed in the present study. Results show that peripheral (membranous) localization of cortactin occurred in 10 out of $20(50 \%)$ patients. The
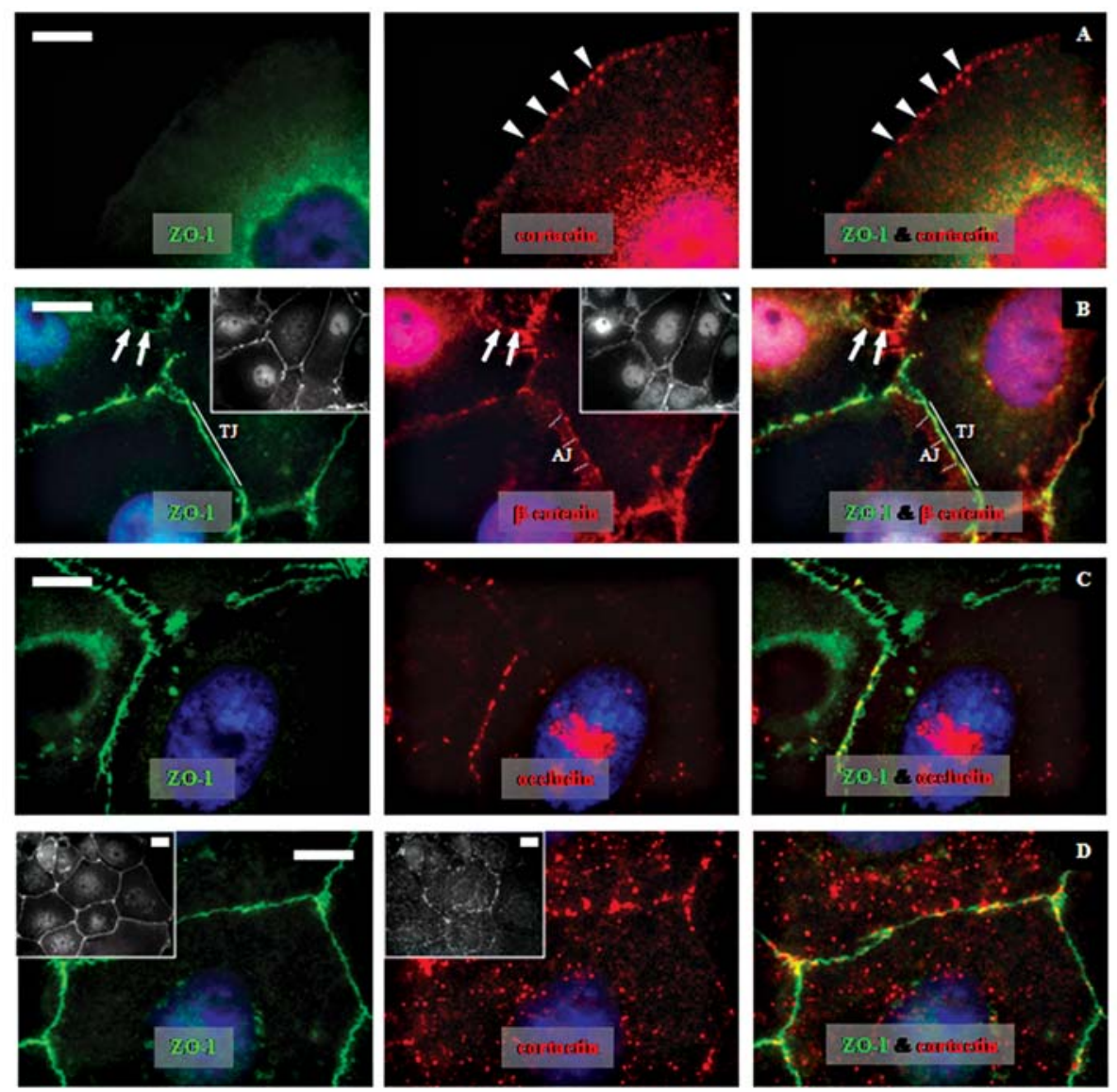

Figure 5. Localization of ZO-1, cortacin, B-catenin and occludin. (A) Double immunofluorescence of ZO-1 (green) and cortacitn (red) in migrating cells. Cortactin was found at leading edge of cells (arrowheads). No accumulation of ZO-1 was found at the leading edge of cells (green, left panel). (B) Double staining of ZO1 (green) and B-catenin (red). Colocalization of ZO-1 and B-catenin was found at primordial cell-cell junction (arrow). TJ, tight junction. AJ, adherence junction. (C) Staining of ZO-1 (green) and occludin (red). Colocalization of ZO-1 and occludin was found in tight junction. Occludin accumulation was not detected at primordial cell-cell junction. (D) Staining of ZO-1 (green) and cortactin (red) in polarized cells. Colocalization of ZO-1 and cortactin was found in cell-cell junction. Bar, $10 \mu \mathrm{m}$. 
Table I. Association between cortactin immunoreactivity and lymph node metastasis.

Lymph node metastasis

$(-)$

$(+)$

\begin{tabular}{lcc}
\hline Total number & 20 & 20 \\
Age & $75.1 \pm 7.5$ & $76.5 \pm 13.1$ \\
Gender $(\mathrm{M} / \mathrm{F})$ & $12 / 8$ & $11 / 9$ \\
Overexpression & 13 & 12 \\
Periphery & 2 & $10^{\mathrm{a}}$ \\
\hline
\end{tabular}

Peripheral localization of cortactin was associated to lymph node metastasis $\left({ }^{\mathrm{a}} \mathrm{p}=0.0138\right)$.

peripheral localization of cortactin was significantly associated with lymph node metastasis $(\mathrm{p}<0.05)$ (Table I and Fig. 3).

Interaction of ZO-1, cortactin and $\beta$-catenin. Co-immunoprecipitation studies showed a direct interaction of $\mathrm{ZO}-1 /$ cortactin and ZO-1/ß-catenin in LS123 cells. Higher interaction of ZO-1 and cortactin was also found in $100 \%$ confluent cells and lower interaction of these molecules in $30 \%$ confluent cells. In contrast, lower interaction of ZO-1 and B-catenin was found in $100 \%$ confluent cells and lower interaction of these molecules was obtained in $30 \%$ confluent cells. In this study, we were not able to co-immunoprecipitate cortactin and B-catenin (Fig. 4).

Immunocytochemistry. Immunocytochemistry studies were conducted to reveal the underlying mechanisms involved in the difference between the interaction of $\mathrm{ZO}-1 /$ cortactin and ZO-1/B-catenin within $30 \%$ confluent cells and $100 \%$ confluent cells. In migrating cells, cortactin was found at the leading edge of cancer cells. In polarized cells, which had TJ, cortactin was observed at cell-cell junction. Double immunofluorescence study showed colocalization of ZO-1 and cortactin at the TJ. Colocalization of ZO-1 and B-catenin was found at primordial cell-cell junction, where tight junction protein occludin was not obtained (Fig. 5).

\section{Discussion}

In this study, we demonstrated that the localization of cortactin at cell periphery was significantly associated with lymph node involvement. We evaluated whether cortactin effects the development of colorectal cancer. Results indicate that the association of ZO-1 and cortactin plays an important role in regulating cell adhesion and spreading.

ZO-1 is a component of cell-cell junction and is thought to work in a structural linkage between cell-cell junctions and cytoskeletal networks (21-23). Cortactin also has an F-actin cross-linking activity and has been implicated in cytoskeleton reorganization (12-14).

Cortactin overexpression is known to be associated with the development of cancer (7-11). The overexpression of cortactin obtained by immunohistochemistry in $62.5 \%$ cases of colorectal cancer is consistent with previous findings. Furthermore, the present results indicate that the overexpression of cortactin and the localization of cortactin complex are critical factors for progression of cancer.

Cancer metastasis involves a series of events that include dissociation of malignant cells from a primary site, migration, intravasation into circulatory system, and adhesion to the vascular endothelium followed by extravasation, invasion, and growth at distant sites (26). It is possible that polarization and depolarization of cancer cells often occur in cancer progression. Thus, in this study, we analyzed $30 \%$ confluent cells, which are considered as migrating, and $100 \%$ confluent cells, which are considered as polarized growth. In initial stages during the formation of cell-cell junctions of epithelial cells, primordial spot-like cell-cell junction is first formed at the tips of the cellular protrusions radiating from adjacent cells (27-30). Cadherin and ZO-1 colocalize to the primordial junction where occludin is not concentrated (28). As cellular polarization proceeds, occludin gradually accumulates at the spot-like junction to form TJ, and cadherin is sorted from occludin and ZO-1 from adherence junction. Consistent with these studies, we found ZO-1 and B-catenin at primordial cell-cell junction. In polarized cells, that were observed in $100 \%$ confluence, cortactin was localized at the cell-cell junction with $\mathrm{ZO}-1$. These results are consistent with previous studies $(24,31)$. Based on these findings we propose that dissociation of ZO-1 and cortactin in cancer cells is critical for cancer cell migration.

ZO-1 accumulation at the leading edge of migrating cells was not observed in the present experiments. In contrast to these results, several investigators have reported the existence of ZO-1 in the leading edge of fibroblasts migrating in a wound and cancer cells, where it has been suggested to initiate integrin-dependent adhesion complexes $(32,33)$. Further studies are needed to clarify the role of ZO-1 in cancer cell adhesion and migration.

In conclusion, the present results showed an association between the localization of cortactin and cancer development. In our previous study, we reported the association between loss of polarity and cancer cell development (25). Together, these findings indicate that the loss of cell-cell adhesion and cell polarity, which is commonly observed in advanced cancer, may be directly involved in cell signaling. Further studies are needed on cell adhesion and signaling for cancer development.

\section{References}

1. Wu H, Reynolds AB, Kanner SB, Vines RR and Parsons JT: Identification and characterization of a novel cytoskeletonassociated pp60src substrate. Mol Cell Biol 11: 5113-5124, 1991.

2. Wu H and Parsons JT: Cortactin, an 80/85-kilodalton pp60src substrate, is a filamentous actin-binding protein enriched in the cell cortex. J Cell Biol 120: 1417-1426, 1993.

3. Schuuring E, Verhoeven E, Litvinov S and Michalides RJ: The product of the EMS1 gene, amplified and overexpressed in human carcinomas, is homologous to a v-src substrate and is located in cell-substratum contact sites. Mol Cell Biol 13: 2891-2898, 1993.

4. Schuuring E, Verhoeven E, Mooi WJ and Michalides RJ: Identification and cloning of two overexpressed genes, U21B31/ PRAD1 and EMS1, within the amplified chromosome 11q13 region in human carcinomas. Oncogene 7: 355-361, 1992. 
5. Weed SA and Parsons JT: Cortactin: coupling membrane dynamics to cortical actin assembly. Oncogene 20: 6418-6434, 2001.

6. Cosen-Binker LI and Kapus A: Cortactin: the gray eminence of the cytoskeleton. Physiology (Bethesda) 21: 352-361, 2006.

7. Chuma M, Sakamoto M, Yasuda J, Fujii G, Nakanishi K, Tsuchiya A, Ohta T, Asaka M and Hirohashi S: Overexpression of cortactin is involved in motility and metastasis of hepatocellular carcinoma. J Hepatol 41: 629-636, 2004

8. Tsai WC, Jin JS, Chang WK, Chan DC, Yeh MK, Cherng SC, Lin LF, Sheu LF and Chao YC: Association of cortactin and fascin-1 expression in gastric adenocarcinoma: correlation with clinicopathological parameters. J Histochem Cytochem 55: 955-962, 2007.

9. Patel AM, Incognito LS, Schechter GL, Wasilenko WJ and Somers KD: Amplification and expression of EMS-1 (cortactin) in head and neck squamous cell carcinoma cell lines. Oncogene 12: 31-35, 1996.

10. Lee YY, Yu CP, Lin CK, Nieh S, Hsu KF, Chiang H and Jin JS: Expression of survivin and cortactin in colorectal adenocarcinoma: association with clinicopathological parameters. Dis Markers 26: 9-18, 2009

11. Rothschild BL, Shim AH, Ammer AG, Kelley LC, Irby KB, Head JA, Chen L, Varella-Garcia M, Sacks PG, Frederick B, Raben D and Weed SA: Cortactin overexpression regulates actin-related protein $2 / 3$ complex activity, motility and invasion in carcinomas with chromosome 11q13 amplification. Cancer Res 66: 8017-8025, 2006.

12. Uruno T, Liu J, Zhang P, Fan Yx, Egile C, Li R, Mueller SC and Zhan X: Activation of Arp2/3 complex-mediated actin polymerization by cortactin. Nat Cell Biol 3: 259-266, 2001.

13. Weaver AM, Heuser JE, Karginov AV, Lee WL, Parsons JT and Cooper JA: Interaction of cortactin and N-WASp with Arp2/3 complex. Curr Biol 12: 1270-1278, 2002.

14. Weed SA, Karginov AV, Schafer DA, Weaver AM, Kinley AW, Cooper JA and Parsons JT: Cortactin localization to sites of actin assembly in lamellipodia requires interactions with F-actin and the Arp2/3 complex. J Cell Biol 151: 29-40, 2000.

15. Machesky LM, Reeves E, Wientjes F, Mattheyse FJ, Grogan A, Totty NF, Burlingame AL, Hsuan JJ and Segal AW: Mammalian actin-related protein $2 / 3$ complex localizes to regions of lamellipodial protrusion and is composed of evolutionarily conserved proteins. Biochem J 328: 105-112, 1997.

16. Huang C, Liu J, Haudenschild CC and Zhan X: The role of tyrosine phosphorylation of cortactin in the locomotion of endothelial cells. J Biol Chem 273: 25770-25776, 1998.

17. Patel AS, Schechter GL, Wasilenko WJ and Somers KD Overexpression of EMS1/cortactin in NIH3T3 fibroblasts causes increased cell motility and invasion in vitro. Oncogene 16 : 3227-3232, 1998 .

18. Li Y, Tondravi M, Liu J, Smith E, Haudenschild CC, Kaczmarek M and Zhan X: Cortactin potentiates bone metastasis of breast cancer cells. Cancer Res 61: 6906-6911, 2001.

19. Itoh M, Nagafuchi A, Yonemura S, Kitani-Yasuda T, Tsukita S and Tsukita S: The 220-kDa protein colocalizing with cadherins in non-epithelial cells is identical to ZO-1, a tight junctionassociated protein in epithelial cells: cDNA cloning and immunoelectron microscopy. J Cell Biol 121: 491-502, 1993.
20. Willott E, Balda MS, Fanning AS, Jameson B, van Itallie C and Anderson JM: The tight junction protein $\mathrm{ZO}-1$ is homologous to the Drosophila discs-large tumor suppressor protein of septate junctions. Proc Natl Acad Sci USA 90: 7834-7838, 1993.

21. Itoh M, Furuse M, Morita K, Kubota K, Saitou M and Tsukita S: Direct binding of three tight junction-associated MAGUKs, ZO-1, ZO-2 and ZO-3, with the $\mathrm{COOH}$ termini of claudins. J Cell Biol 147: 1351-1363, 1999.

22. Fanning AS, Jameson BJ, Jesaitis LA and Anderson JM: The tight junction protein ZO-1 establishes a link between the transmembrane protein occludin and the actin cytoskeleton. J Biol Chem 273: 29745-29753, 1998.

23. Itoh M, Nagafuchi A, Moroi S and Tsukita S: Involvement of ZO-1 in cadherin-based cell adhesion through its direct binding to alpha catenin and actin filaments. J Cell Biol 138: 181-192, 1997.

24. Katsube T, Takahisa M, Ueda R, Hashimoto N, Kobayashi M and Togashi S: Cortactin associates with the cell-cell junction protein ZO-1 in both Drosophila and mouse. J Biol Chem 273: 29672-29677, 1998.

25. Hirakawa H, Nakayama T, Shibata K and Sekine I: Association of cellular localization of glycogen synthase kinase 3beta in the digestive tract with cancer development. Oncol Rep 22: 481-485, 2009.

26. Woodhouse EC, Chuaqui RF and Liotta LA: General mechanisms of metastasis. Cancer 80: 1529-1537, 1997.

27. Yonemura S, Itoh M, Nagafuchi A and Tsukita S: Cell-to-cell adherens junction formation and actin filament organization: similarities and differences between non-polarized fibroblasts and polarized epithelial cells. J Cell Sci 108: 127-142, 1995.

28. Ando-Akatsuka Y, Yonemura S, Itoh M, Furuse M and Tsukita S: Differential behavior of E-cadherin and occludin in their colocalization with ZO-1 during the establishment of epithelial cell polarity. J Cell Physiol 179: 115-125, 1999.

29. Adams CL, Chen YT, Smith SJ and Nelson WJ: Mechanisms of epithelial cell-cell adhesion and cell compaction revealed by high-resolution tracking of E-cadherin-green fluorescent protein. J Cell Biol 142: 1105-1119, 1998.

30. Vasioukhin V, Bauer C, Yin M and Fuchs E: Directed actin polymerization is the driving force for epithelial cell-cell adhesion. Cell 100: 209-219, 2000

31. Wu H and Montone KT: Cortactin localization in actin-containing adult and fetal tissues. J Histochem Cytochem 46: 1189-1191, 1998.

32. Taliana L, Benezra M, Greenberg RS, Masur SK and Bernstein AM: ZO-1: lamellipodial localization in a corneal fibroblast wound model. Invest Ophthalmol Vis Sci 46: 96-103, 2005.

33. Tuomi S, Mai A, Nevo J, Laine JO, Vilkki V, Ohman TJ, Gahmberg CG, Parker PJ and Ivaska J: PKCepsilon regulation of an alpha5 integrin-ZO-1 complex controls lamellae formation in migrating cancer cells. Sci Signal 2: ra32, 2009. 